\title{
New Synthetic Routes for Ruthenium-1,10-Phenanthroline \\ Complexes. Tests of Cytotoxic and Antibacterial Activity of Selected Ruthenium Complexes.
}

\author{
Iztok Turel $^{a^{*}}$, Amalija Golobič ${ }^{\mathrm{a}}$, Jakob Kljun ${ }^{\mathrm{a}}$, Petra Samastur ${ }^{\mathrm{a}}$, Urška Batista $^{\mathrm{b}}$, Kristina \\ Sepčićc
}

Dedicated to the memory of Prof. Dr. Jurij V. Brenčič.
${ }^{a}$ Faculty of Chemistry and Chemical Technology, University of Ljubljana, Večna pot 113, SI- 1000 Ljubljana, Slovenia.
${ }^{b}$ Institute of Biophysics, Faculty of Medicine, University of Ljubljana, Lipičeva 2, 1000 Ljubljana, Slovenia
${ }^{\mathrm{c}}$ Biotechnical Faculty, Department of Biology, University of Ljubljana, Večna pot 111, 1000 Ljubljana, Slovenia.
*To whom correspondence should be addressed: Tel: +386 1 4798525; E-mail: iztok.turel@fkkt.uni-lj.si

Diffraction data for all three compounds were collected on a Nonius Kappa CCD diffractometer with graphite monochromated $\mathrm{MoK} \alpha$ radiation at temperature $150 \mathrm{~K}$. The data were processed using DENZO ${ }^{1}$ program. Structures were solved by direct methods using SIR $97^{2}$. Most of the positions of hydrogen atoms were obtained from the difference Fourier maps, the remaining were calculated. We employed full-matrix least-squares refinements on $\mathrm{F}$ magnitudes with anisotropic displacement factors for non-hydrogen atoms using Xtal3. ${ }^{3}$. The exception were $\mathrm{C}$ and $\mathrm{O}$ atoms of solvate methanol molecules in $\mathbf{3}$, which are slightly disordered and are thus refined with isotropic displacement parameter. The position of hydroxyl $\mathrm{H}$ atoms of these molecules were not determined. One water molecule (O5W) in $\mathbf{1}$ is disordered over two positions O5W1 and O5W2. These two maxima were also refined with isotropic displacement parameter and the corresponding $\mathrm{H}$ atoms positions were not determined. The parameters of hydrogen atoms were not refined. In the final cycle of the refinement we used 3939, 7201 and 6147 reflections and 217, 397 and 454 parameters for compounds $1,2 \cdot 4 \mathrm{H}_{2} \mathrm{O}$ and $3 \cdot 6 \mathrm{MeOH}$, respectively. The final $R$ values were $0.033,0.030$ and 0.068 and $R_{\mathcal{W}}$ values were $0.030,0.030$ and 0.045 for $\mathbf{1}, \mathbf{2} \cdot 4 \mathrm{H}_{2} \mathrm{O}$ and $\mathbf{3} \cdot 6 \mathrm{MeOH}$ respectively. The crystallographic data were deposited in the Cambridge Crystallographic Data Center and were assigned the deposition numbers CCDC 1028368-1028370 for compounds 1-3 respectively.

\section{References}

1. Z. Otwinowski, W. Minor, Methods Enzymol. 276 (1997) 307. 
2. Altomare, M.C. Burla, M. Camalli, G. Cascarano, C. Giacovazzo, A. Guagliardi, A.G.G. Moliterni, G. Polidori, R. Spagna, J. Appl. Cryst. 32 (1999) 115.

3. Hall, S.R., du Boulay, D.J. \& Olthof-Hazekamp, R. (1999). Eds. Xtal3.6 System . University of Western Australia.

Table S1: Crystallographic data for compounds 1, 2 $4 \mathrm{H}_{2} \mathrm{O}$ and $3 \cdot 6 \mathrm{MeOH}$.

\begin{tabular}{lccc}
\hline \hline & $\mathbf{1}$ & $\mathbf{2} \cdot 4 \mathrm{H}_{2} \mathrm{O}$ & $\mathbf{3} \cdot 6 \mathrm{MeOH}$ \\
\hline Empirical formula & $\mathrm{C}_{14} \mathrm{H}_{14} \mathrm{Cl}_{3} \mathrm{~N}_{2} \mathrm{ORuS}$ & $\mathrm{C}_{12} \mathrm{H}_{19} \mathrm{Cl}_{4} \mathrm{~N}_{2} \mathrm{O}_{5} \mathrm{Ru}$ & $\mathrm{C}_{42} \mathrm{H}_{48} \mathrm{Cl}_{2} \mathrm{~N}_{6} \mathrm{O}_{6} \mathrm{Ru}$ \\
$\mathrm{M}_{\mathrm{w}}$ & 465.76 & 514.16 & 904.858 \\
$T, \mathrm{~K}$ & $150(2)$ & $150(2)$ & $150(2)$ \\
Crystal system & triclinic & triclinic & orthorhombic \\
Space group & $P-1$ & $P-1$ & $P b c a$ \\
$a, \AA$ & $8.8592(1)$ & $8.1409(2)$ & $18.5607(3)$ \\
$b, \AA$ & $11.7145(2)$ & $9.2715(2)$ & $18.6235(3)$ \\
$c, \AA$ & $16.7731(3)$ & $13.0919(3)$ & $23.7095(5)$ \\
$\alpha$, deg. & $92.5915(7)$ & $86.061(1)$ & 90.000 \\
$\beta$, deg. & $103.1136(7)$ & $77.551(1)$ & 90.000 \\
$\gamma$, deg. & $93.3115(7)$ & $78.652(1)$ & 90.000 \\
$V, \AA^{3}$ & $1689.46(5)$ & $945.70(4)$ & $8195.5(3)$ \\
$Z$ & 4 & 2 & 8 \\
$D_{\text {calc }}, \mathrm{g} / \mathrm{cm}{ }^{3}$ & 1.831 & 1.806 & 1.466 \\
$\mu$, mm & 1.527 & 1.418 & 0.568 \\
$F(000)$ & 924 & 514 & 3672 \\
Crystal size, mm & $0.44 \times 0.16 \times 0.14$ & $0.175 \times 0.125 \times 0.10$ & $0.30 \times 0.25 \times 0.20$ \\
Color & red & brown & red \\
Data collected / unique & $24614 / 7691$ & $16369 / 4297$ & $30130 / 12959$ \\
$\mathrm{R}_{\text {int }}$ & 0.031 & 0.039 & 0.10 \\
Restraints / parameters & $0 / 397$ & $0 / 217$ & $0 / 454$ \\
$\mathrm{~S}$ & 1.218 & 1.089 & 1.100 \\
$R_{l}, w R_{2}[\mathrm{I}>2 \sigma(\mathrm{I})]$ & $0.030 / 0.030$ & $0.033 / 0.030$ & $0.068 / 0.045$ \\
$R_{l}, w R_{2}($ all data) & $0.043 / 0.056$ & $0.058 / 0.067$ & $0.197 / 0.085$ \\
Largest diff. peak / hole $\left(\mathrm{e} \AA^{-3}\right)$ & $0.749 /-0.962$ & $0.792 /-0.614$ & $1.857 /-3.293 *$ \\
\hline \hline
\end{tabular}

*The largest diff. peak and hole in $\mathbf{3}$ is located in close proximity to the central ruthenium ion 
Table S2: Selected geometric parameters $\left(\AA,{ }^{\circ}\right)$ in $\mathbf{1}$.

\begin{tabular}{cccc}
\hline \hline Ru1a-Cl1a & $2.3528(14)$ & Ru1b-N2b & $2.093(5)$ \\
\hline Ru1a-Cl2a & $2.3512(13)$ & Ru1b-Cl3b & $2.3350(12)$ \\
\hline Ru1a-Cl3a & $2.3346(12)$ & Ru1b-Cl1b & $2.3333(13)$ \\
\hline Ru1a-S1a & $2.2935(12)$ & Ru1b-Cl2b & $2.3461(13)$ \\
\hline Ru1a-N1a & $2.086(4)$ & Ru1b-S1b & $2.3114(13)$ \\
\hline Ru1a-N2a & $2.097(5)$ & Ru1b-N1b & $2.102(5)$ \\
\hline Cl1a-Ru1a-Cl2a & $91.97(5)$ & Cl1b-Ru1b-N1b & $85.79(13)$ \\
\hline Cl1a-Ru1a-Cl3a & $92.93(5)$ & Cl1b-Ru1b-N2b & $88.21(13)$ \\
\hline Cl1a-Ru1a-S1a & $86.62(5)$ & Cl2b-Ru1b-Cl3b & $95.18(5)$ \\
\hline Cl1a-Ru1a-N1a & $174.11(13)$ & Cl2b-Ru1b-S1b & $89.61(5)$ \\
\hline Cl1a-Ru1a-N2a & $94.74(13)$ & Cl2b-Ru1b-N1b & $86.93(13)$ \\
\hline Cl2a-Ru1a-Cl3a & $174.42(5)$ & Cl2b-Ru1b-N2b & $89.04(13)$ \\
\hline Cl2a-Ru1a-S1a & $92.39(4)$ & Cl3b-Ru1b-S1b & $87.22(4)$ \\
\hline Cl2a-Ru1a-N1a & $86.83(11)$ & Cl3b-Ru1b-N1b & $172.19(13)$ \\
\hline Cl2a-Ru1a-N2a & $92.18(13)$ & Cl3b-Ru1b-N2b & $93.34(14)$ \\
\hline Cl3a-Ru1a-S1a & $90.55(4)$ & S1b-Ru1b-N1b & $100.33(13)$ \\
\hline Cl3a-Ru1a-N1a & $88.03(11)$ & S1b-Ru1b-N2b & $178.58(13)$ \\
\hline Cl3a-Ru1a-N2a & $84.77(13)$ & N1b-Ru1b-N2b & $79.16(18)$ \\
\hline S1a-Ru1a-N1a & $99.19(12)$ & Cl1b-Ru1b-Cl3b & $91.85(4)$ \\
\hline S1a-Ru1a-N2a & $175.18(14)$ & Cl1b-Ru1b-S1b & $93.08(5)$ \\
\hline N1a-Ru1a-N2a & $79.56(17)$ & Cl1b-Ru1b-Cl2b & $172.59(5)$ \\
\hline \hline
\end{tabular}

Table S3: Selected geometric parameters $\left(\AA,{ }^{\circ}\right)$ in $\mathbf{2} \cdot 4 \mathrm{H}_{2} \mathrm{O}$.

\begin{tabular}{cccc}
\hline \hline $\mathrm{Ru}-\mathrm{Cl} 1$ & $2.3483(16)$ & $\mathrm{Ru}-\mathrm{N} 2$ & $2.056(5)$ \\
\hline $\mathrm{Ru}-\mathrm{Cl} 2$ & $2.3490(16)$ & $\mathrm{N} 1-\mathrm{C} 10$ & $1.377(7)$ \\
\hline $\mathrm{Ru}-\mathrm{Cl} 3$ & $2.3890(16)$ & $\mathrm{N} 1-\mathrm{C} 2$ & $1.330(8)$ \\
\hline $\mathrm{Ru}-\mathrm{Cl} 4$ & $2.3854(15)$ & $\mathrm{N} 2-\mathrm{C} 9$ & $1.331(8)$ \\
\hline $\mathrm{Ru}-\mathrm{N} 1$ & $2.064(5)$ & $\mathrm{N} 2-\mathrm{C} 12$ & $1.365(8)$ \\
\hline $\mathrm{Cl} 1-\mathrm{Ru}-\mathrm{Cl} 2$ & $177.28(5)$ & $\mathrm{Cl}-\mathrm{Ru}-\mathrm{N} 2$ & $91.46(14)$ \\
\hline $\mathrm{Cl1}-\mathrm{Ru}-\mathrm{Cl} 3$ & $88.89(6)$ & $\mathrm{Cl}-\mathrm{Ru}-\mathrm{Cl} 4$ & $89.60(6)$ \\
\hline $\mathrm{Cl} 1-\mathrm{Ru}-\mathrm{Cl} 4$ & $91.17(5)$ & $\mathrm{Cl}-\mathrm{Ru}-\mathrm{N} 1$ & $95.66(14)$ \\
\hline $\mathrm{C} 11-\mathrm{Ru}-\mathrm{N} 1$ & $89.81(14)$ & $\mathrm{Cl}-\mathrm{Ru}-\mathrm{N} 2$ & $174.78(15)$ \\
\hline $\mathrm{C} 11-\mathrm{Ru}-\mathrm{N} 2$ & $87.85(14)$ & $\mathrm{Cl}-\mathrm{Ru}-\mathrm{N} 1$ & $174.67(14)$ \\
\hline $\mathrm{Cl} 1-\mathrm{Ru}-\mathrm{Cl} 3$ & $91.61(6)$ & $\mathrm{Cl} 1-\mathrm{Ru}-\mathrm{N} 2$ & $94.54(15)$ \\
\hline $\mathrm{Cl} 2-\mathrm{Ru}-\mathrm{Cl} 1$ & $91.51(5)$ & $\mathrm{N} 1-\mathrm{Ru}-\mathrm{N} 2$ & $80.26(19)$ \\
\hline $\mathrm{Cl} 2-\mathrm{Ru}-\mathrm{N} 1$ & $87.48(14)$ & & \\
\hline \hline
\end{tabular}

Table S4: Selected geometric parameters $\left(\AA,^{\circ}\right)$ in $\mathbf{3} \cdot 6 \mathrm{MeOH}$.

\begin{tabular}{cccc}
\hline \hline Ru-N1a & $2.063(8)$ & $\mathrm{Ru}-\mathrm{N} 10 \mathrm{a}$ & $2.075(9)$ \\
\hline $\mathrm{Ru}-\mathrm{N} 1 \mathrm{~b}$ & $2.071(9)$ & $\mathrm{Ru}-\mathrm{N} 10 \mathrm{~b}$ & $2.065(9)$ \\
\hline $\mathrm{Ru}-\mathrm{N} 1 \mathrm{c}$ & $2.067(7)$ & $\mathrm{Ru}-\mathrm{N} 10 \mathrm{c}$ & $2.066(9)$ \\
\hline $\mathrm{N} 1 \mathrm{a}-\mathrm{Ru}-\mathrm{N} 1 \mathrm{~b}$ & $96.6(3)$ & $\mathrm{N} 1 \mathrm{a}-\mathrm{Ru}-\mathrm{N} 10 \mathrm{c}$ & $88.2(3)$ \\
\hline $\mathrm{N} 1 \mathrm{a}-\mathrm{Ru}-\mathrm{N} 1 \mathrm{c}$ & $93.0(3)$ & $\mathrm{N} 1 \mathrm{~b}-\mathrm{Ru}-\mathrm{N} 1 \mathrm{c}$ & $94.9(3)$ \\
\hline $\mathrm{N} 1 \mathrm{a}-\mathrm{Ru}-\mathrm{N} 10 \mathrm{a}$ & $80.3(4)$ & $\mathrm{N} 1 \mathrm{~b}-\mathrm{Ru}-\mathrm{N} 10 \mathrm{a}$ & $90.9(3)$ \\
\hline $\mathrm{N} 1 \mathrm{a}-\mathrm{Ru}-\mathrm{N} 10 \mathrm{~b}$ & $173.9(3)$ & $\mathrm{N} 1 \mathrm{~b}-\mathrm{Ru}-\mathrm{N} 10 \mathrm{~b}$ & $80.3(3)$ \\
\hline \hline
\end{tabular}


Table S5: Hydrogen bond contact distances and angles in compound $2 \cdot 4 \mathrm{H}_{2} \mathrm{O}$. $\mathrm{O} 5 \mathrm{w} 1$ and $\mathrm{O} 5 \mathrm{w} 2$ atoms have $50 \%$ occupancy.

\begin{tabular}{|c|c|c|c|}
\hline Donor (D) & Acceptor (A) & D...A $(\AA)$ & D-H...A $\left(^{\circ}\right)$ \\
\hline $\mathrm{O} 1 \mathrm{w}$ & $03 \mathrm{w}^{1-\mathrm{x}, 1-\mathrm{y}, 2-\mathrm{z}}$ & $2.642(7)$ & 165 \\
\hline $\mathrm{O} 1 \mathrm{w}$ & $\mathrm{O} 4 \mathrm{w}$ & $2.432(7)$ & 174 \\
\hline O1w & $\mathrm{O} 5 \mathrm{w} 1^{-x, 2-\mathrm{y}, 2-\mathrm{z}}$ & $2.610(10)$ & 174 \\
\hline O1w & $\mathrm{O} 5 \mathrm{w} 2^{-\mathrm{x}, 2-\mathrm{y}, 2-\mathrm{z}}$ & $2.467(16)$ & 154 \\
\hline $\mathrm{O} 2 \mathrm{w}$ & $\mathrm{Cl} 14^{-\mathrm{x}, 2-\mathrm{y}, 2-\mathrm{z}}$ & $3.222(5)$ & 163 \\
\hline $\mathrm{O} 2 \mathrm{w}$ & $\mathrm{C} 13^{\mathrm{x}, 1+\mathrm{y}, \mathrm{z}}$ & $3.420(4)$ & 162 \\
\hline $\mathrm{O} 3 \mathrm{w}$ & $\mathrm{Cl} 3^{1+\mathrm{x}, \mathrm{y}, \mathrm{z}}$ & $3.202(5)$ & 164 \\
\hline $\mathrm{O} 3 \mathrm{w}$ & $\mathrm{Cl} 2$ & $3.108(5)$ & 166 \\
\hline $\mathrm{O} 4 \mathrm{w}$ & $\mathrm{O} 3 \mathrm{w}$ & $2.737(7)$ & 178 \\
\hline $\mathrm{O} 4 \mathrm{w}$ & $\mathrm{O} 2 \mathrm{w}^{1-\mathrm{I}, 2-\mathrm{y}, 2-\mathrm{z}}$ & $2.725(7)$ & 175 \\
\hline O5w1 & $\mathrm{O} 2 \mathrm{w}$ & $2.791(12)$ & - \\
\hline O5w1 & $\mathrm{Cl1}$ & $3.130(15)$ & - \\
\hline O5w2 & $\mathrm{O} 2 \mathrm{w}$ & $2.711(17)$ & - \\
\hline O5w2 & $\mathrm{Cl1}$ & $3.186(11)$ & - \\
\hline
\end{tabular}


Table S6: Minimal inhibitory concentrations (MIC) of the tested compounds against selected bacteria. n.i. $=$ no inhibition.

\begin{tabular}{|l|c|c|c|c|c|c|}
\hline \multicolumn{7}{|c|}{ MIC (ug/ml) } \\
\hline Microorganism & $\mathbf{1}$ & $\mathbf{4}$ & $\mathbf{5}$ & $\mathbf{P}$ & phen & Acv \\
\hline Klebsiella pneumoniae & 800 & $>1000$ & $>1000$ & $>1000$ & 60 & $>1000$ \\
\hline Staphylococcus aureus & 250 & $>1000$ & $>1000$ & $>1000$ & 20 & $>1000$ \\
\hline Escherichia coli & 500 & $>1000$ & $>1000$ & $>1000$ & 75 & $>1000$ \\
\hline Salmonella typhimurium & 1000 & $>1000$ & $>1000$ & $>1000$ & 50 & $>1000$ \\
\hline Proteus vulgaris & 900 & $>1000$ & $>1000$ & $>1000$ & 35 & $>1000$ \\
\hline Pseudomonas aeruginosa & $>1000$ & $>1000$ & $>1000$ & $>1000$ & $>1000$ & $>1000$ \\
\hline Micrococcus luteus & $>1000$ & $>1000$ & $>1000$ & $>1000$ & 250 & $>1000$ \\
\hline Bacillus subtilis & $>1000$ & $>1000$ & $>1000$ & $>1000$ & 250 & $>1000$ \\
\hline
\end{tabular}

